International Conference on New Interfaces for Musical Expression

\title{
NIME and the
}

Environment: Toward a

More Sustainable NIME

\section{Practice}

Raul Masu' ${ }^{1}$, Adam Pultz Melbye ${ }^{2}$, John Sullivan ${ }^{3}$, Alexander Refsum Jensenius ${ }^{4}$

${ }^{1}$ DI, FCT, Universidade NOVA de Lisboa, ITI/LARSyS, and NOVA Lincs,

${ }^{2}$ Sonic Arts Research Centre, Queen's University, Belfast,

${ }^{3}$ IDMIL, CIRMMT. MCGill University. Montréal, Canada,

${ }^{4}$ University of Oslo, Department of Musicology, RITMO Centre for Interdisciplinary Studies in Rhythm, Time, and Motion

License: Creative Commons Attribution 4.0 International License (CC-BY 4.0). 


\begin{abstract}
This paper addresses environmental issues around NIME research and practice. We discuss the formulation of an environmental statement for the conference as well as the initiation of a NIME Eco Wiki containing information on environmental concerns related to the creation of new musical instruments. We outline a number of these concerns and, by systematically reviewing the proceedings of all previous NIME conferences, identify a general lack of reflection on the environmental impact of the research undertaken. Finally, we propose a framework for addressing the making, testing, using, and disposal of NIMEs in the hope that sustainability may become a central concern to researchers.
\end{abstract}

\title{
Author Keywords
}

NIME, Environmental Impact, Wikis, Digital Commons

\section{CCS Concepts}

-Human-centered computing $\rightarrow$ Collaborative and social computing $\rightarrow$ Collaborative and social computing systems and tools.

\section{Introduction}

Environmental issues have been discussed for decades, yet the topic has received remarkably little attention in the NIME community. The authors first met as part of an initiative to develop an environmental statement for the NIME conference. The statement, published on the nime.org website, serves as a policy document both within and outside of the community. The work on the environmental statement sparked an interest in investigating the topic further. In this paper, we report on the current state of affairs concerning sustainable NIME practice, from individual to the community. The first aim was to review the NIME literature for papers related to environmental issues or practices. Next, we focused on developing the NIME Eco Wiki, including suggestions and tools to help NIME researchers and practitioners develop more environmentally-friendly practices. Finally, we propose a model for the systematic inclusion of environmental action in NIME research practice.

\section{Background}




\section{NIME Environmental Statement}

To promote awareness of environmental issues in the community, the NIME Conference Environmental Statement is a policy document outlining the bigger picture and making a call for action. It was developed during the fall of 2020 through a series of online workshops and collaborative writing. The statement was endorsed by the NIME Steering Committee in November 2020 and was ultimately launched on nime.org. The short version of the statement reads:

Every action we perform, including research, has an impact on our ecosystem. NIME is committed to environmental sustainability and conservation in both the delivery of the annual conference and in our day to day research and artistic practices. The complete NIME Conference Environmental Statement can be found at: www.nime.org/environment.

The statement is thought of as a living document, owned by the community. It is released with a CC-BY license, to promote sharing of the content, for example, to other conferences and communities.

\section{(Self) reflection in the NIME community}

In recent years, a tradition of auto-analysis has emerged in the NIME conference. For instance, Marquez-Borbon and Stapleton reflected on the NIME community's values, addressing the positive effect of structuring knowledge in documented activities to help guide beginners' practice and learning process. They also reflected on how the lack of community understanding of performance inhibits constructive critique development [1]. Discussing the meaning of the community, Cantrell proposed five broad cultural categories for looking at histories, as well as creative and technical discourses surrounding the design of new musical instruments [2]. Other reflections on the community focused on what craft means within the NIME community [ $\underline{3}$ ].

Surveys proved to be of use, for instance, to investigate "control, instrumental entropy, limitations, and creativity" in NIMEs [4]], to study the use of thematic analysis approach as input to the design of new DMIs [ㅁ] , and to look at the identity on NIMEs concerning the aesthetic of the music and the repertoire []].

Systematic analysis of NIME proceedings has been conducted to study the meaning of the word gesture for NIME authors []], evaluation strategies used (or not used) []], and to look for trends in the development of NIMEs [9] . In [10], authors analyzed proceedings from the NIME, ICMC, and SMC conferences to explore how issues 
related to digital musical instrument (DMI) stability, reliability, and compatibility have been addressed in prior research.

\section{Ecologies and ethics}

The term ecology has mainly been used in the discourse on DMIs to indicate the entire complex set of agents, including performers, composers, other instruments, environment, etc. For instance, Gurevich and Trevino proposed that the ecology of music creation should focus on the "relationships between composers, performers, and listeners as a part of a system" that also considers history, genre, and context [11]. Similarly, Waters speaks about performance ecosystems to study the interaction between performers, instruments, and environments [12]. Recently, this perspective was further developed by borrowing the concept of artefact ecology [13],[14],[15] or reflecting on the quality of musical instruments [16]. However, the idea of ecology in an environmental sense is still under-explored.

Only recently, a few authors have started to reflect on the political implications of NIME research, and in one case, directly pointing out environmental issues. Hayes and Marquez-Borbon mainly focused on diversity, inclusion, and accessibility while also addressing the market demands of the neoliberal university [17]. Morreale et al. open up the perspective by arguing for the need for an outward-looking effort that accounts for social, ethical, and cultural issues related to new digital musical instruments created within and outside the NIME discourse [18]. In their paper, Morreale et al. also touch on environmental issues:

"environmental costs are not limited to the production and disposal phase, and our analysis should also include environmental costs at run time" [18].

\section{Intersection of NIME practice/music technology and environmental issues}

\section{Physical impact}

As with all other technologies, musical instrument making was initially dependent on local resources such as when Stradivari sourced materials from the woods of Paneveggio. However, with the rise of imperialism and increased international trade in the 17th and 18th century, ivory became common for piano keys from around 1700 [19] - a practice that was only banned in 1989 under the CITES (Convention on International Trade in Endangered Species of Wild Fauna and Flora) treaty [20]. Brazilian Rosewood, used extensively in guitar making up until the 1960s, is similarly 
banned under CITES [21]. While NIMEs rarely, if ever, make use of exotic woods, let alone elephant tusk, their heavy reliance on electrical and digital technologies suggests that other factors may be at play in an evaluation of the sustainability of its designs. Devine [22] brought attention to the difficulty of identifying the raw material supply chain for the PVC and resin used in vinyl record production. Likely, the supply chain for the basic electronic components of a DMI is just as opaque, possibly a reason why this is rarely addressed in the literature. An exception is Collins where the author expresses "The desire to shrink my silicon footprint "[23]. While Collins' concern here is with the environmental impact of electronic recycling in China, a byproduct of silicon extraction is carbon monoxide, putting the miners involved with its extraction at risk of developing silicosis [24]

On the industry level, companies such as Fairphone address these issues by releasing products with interchangeable parts that can be repaired and safely recycled, while using minerals that are as much as possible sustainably mined. These are bold claims and the validity of which will be beyond this paper's scope to investigate. Individual NIMEs are usually produced in low numbers (see [ㅁ]) and as such do not directly engage with mass production in their output phase. It is, however, a concern that the input phase of the designs relies on mass-produced electronics, the production and disposal of which have global environmental consequences. Additionally, with a core principle of the community being research and experimentation, the sometimes multiple iterations leading to a working NIME are in danger of creating a trail of electronic scrap, some of which will be unusable for new designs. As such, the environmental impact of a NIME may not be evident from the mere observation of its most recent iteration.

Significantly, the sharp fall in 3D-printing costs has made certain kinds of prototyping much more straightforward, leading to the risk of proliferation of intermediate iterations and hence, an increase in PLA waste. PLA, being a bioplastic, requires an industrial composter for its recycling [25] and as such, requires a rigorous infrastructure for its secure disposal. Additionally, PLA is made from (often genetically modified) corn and therefore presents the same problems as biofuels: monoculture farming that impoverishes topsoil and requires fertiliser and pesticides while occupying land where food could be grown, ultimately threatening biodiversity [26].

\section{Data impact}

As with virtually all other areas of life, machine learning has become ubiquitous in music technology, for example, in Izotope's RX and Neutron software [27]. While 
machine learning is often used in NIMEs, such as the Wekinator [28], their algorithms are built to train and run on laptop computers (and in the case of Wekinator, in realtime). As such, they are nowhere near as energy-hungry as large-scale training networks such as Natural Language Processing algorithms [29]. However, the fact remains that CPU-intensive, and hence, power-hungry processes not exclusive to machine learning have become part of NIME's identity [이] . With the introduction of microprocessors such as Raspberry PI and the Bela, some energy efficiency has been gained, with these processors requiring less power than a laptop and audio interface. However, it is an open question to which extent this gain is enough to offset the environmental impact of the manufacturing and eventual disposal of the microprocessors.

\section{Use and reuse of NIMEs}

A recurring theme brought up in NIME papers is the longevity of DMIs and NIMEs [31] [32][33]. A DMI that ends up on a shelf with no life beyond the initial presentation freezes up resources from a sustainability perspective. We recognise that the individual DMI may form the basis of subsequent DMIs or in other ways inform research or create musical value in the world of instrument design. Still, we agree with Morreale et al. that it is necessary to critically address the high turnout of prototype NIMEs as a cause for environmental concern. Academic and artistic communities are not immune to the capitalist mantra of growth and continuous output. With NIME's research output being directly tied to production chains relying on fossil fuels and mining, it becomes apparent that $\underline{\text { Reduce, }}$ Reuse, Recycle may be a more fitting mantra.

\section{Systematic review of NIME literature}

To assess the degree to which environmental issues have been raised within NIME, we conducted a review of the NIME conference proceedings. The full proceedings are freely available from the NIME website and are comprised of 1867 papers spanning each year of the conference from 2001 to 2020. The review entailed an automated search for a selection of relevant keywords followed by manual inspection of the most relevant papers.

The keyword search of the proceedings utilises the OS X terminal command mdfind to return files matching a given query. This technique was used previously in systematic reviews by [7] and [10]. A shell script was written to automate the search and save results in a readable .csv format. $\underline{1}$ The script is placed in the root of a directory 
alongside sub-directories of NIME proceedings by year. It is run from the command line with any number of keywords to be searched for listed as arguments. The results provide the number and filenames of papers containing each keyword for each year.

Several keywords were generated that we felt would likely be mentioned in papers related to environmental topics. After review and discussion, nine terms were selected and run in the search.

\section{Results}

The following table shows each keyword and the number of papers that the term occurred in.

\begin{tabular}{|l|l|}
\hline Keyword & Number of papers \\
\hline environment & 1144 \\
\hline ecology & 68 \\
\hline carbon & 45 \\
\hline footprint & 34 \\
\hline sustainability & 17 \\
\hline climate & 14 \\
\hline pollution & 11 \\
\hline conservation & 4 \\
\hline biodiversity & 2 \\
\hline
\end{tabular}

Unsurprisingly, several of the chosen keywords are used throughout the NIME proceedings in contexts that are unrelated to environmental sustainability. Most notably, environment appears in more than half of all papers. To narrow the results to a smaller subset of papers for manual inspection, only papers in which 2 or more keywords appeared were selected, yielding 124 results.

Number of keywords

Number of papers
Status 


\begin{tabular}{|l|l|l|}
\hline 5 & 1 & keep \\
\hline 4 & 4 & keep \\
\hline 3 & 10 & keep \\
\hline 2 (not including environment) & 1 & keep \\
\hline 2 (including environment) & 108 & keep \\
\hline 1 & 1072 & discard \\
\hline 0 & 671 & discard \\
\hline
\end{tabular}

While this selection method isn't infallible, we found it effective as a filter to reduce the large proceedings to a manageable subset for closer inspection. Here, we found 7 papers that directly engage with environmental concerns, 6 of which present a new NIME, while a further 5 papers mention these concerns in passing, without identifying them as central to the research.

From these 12 papers, we have identified three main concerns:

- Raising awareness of ecological and environmental issues

- Addressing environmental impact of music technology design

- Discussing ecological and environmental concerns within the context of political and cultural issues

In the following, we locate the place of each paper within these categories:

\section{Raising awareness of ecological and environmental issues}

Sonification of data tied to climate change and pollution is found in 6 papers, of which

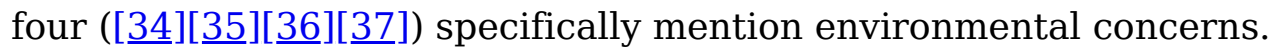

Suchánek [34] describes the sonification of organic material (soil) in reference to drought caused by climate change. According to the author, the installation serves to "communicate an actual environmental topic in a quite unexpected way".

A recent paper by Magnusson et al. [ㅌ5] mentions Eldridge's current reflection about the role of interactive music in "perceptualizing" audio data sets to support understanding and interpretation of environmental data. In particular, the ecoacoustic work, in collaboration with conservation biologists (so also the term conservation is 
used in an ecological-related context), investigates strategies to monitoring biodiversity from Ecuadorian Amazon and cloud forests, Indonesian reefs, and the Swedish Arctic.

In a paper by Lynch and Paradiso [ㅌ6], the term environment is used in many ways, including a reflection on acoustic ecology environments, in connection with literature discussing sounds of trees, and sound in relation to physical/ecological environment. The system presented in the paper can sonify various data, including climate change, daily cycles and weather patterns.

In a paper by Smallwood about sound art [37], environmental interaction through photovoltaic panels is explicitly linked to energy factors, and therefore to environmental awareness. "Various ecological threads of sustainability, including music's own acoustic ecology phenomenon" are mentioned as a background motivation of the work.

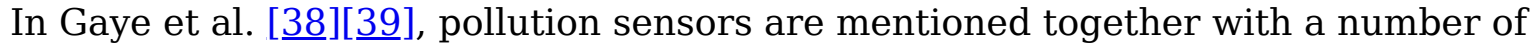
other sensors. However, the authors do not mention environmental concerns.

In discussing an instrument made of discarded wood (driftwood), Rieger and Topel note that our "actions intervene directly with issues relating to sustainability and the amount of value we place on the world in which we live" [40].

\section{Addressing environmental impact of music technology design}

In a paper about Sustainable Instrument Redesign [41], Freed suggests that "the Fingerphone can serve as a strong signal that more environmentally responsible materials and design techniques are available". The carbon footprint of a previously commercially available instrument is mentioned, and the new design is focused on having a low carbon footprint.

Koelhy et al., in a paper on home-made touch sensors, conclude that "these interfaces can even be granted an environmental-friendly label as one can use only recycled materials to produce them" [42].

Fraietta briefly mentions the environmental impact of OSC as compared to MIDI [43]. 


\section{Discussing ecological and environmental concerns within the context of political and cultural issues}

Papers by Morreale et al. [17] and Lepri and McPherson [44] both address or mention sustainability in the context of moral, political and social values. While the data set is too small to draw any definitive conclusions, it may still be worth noticing that we have only found such prescriptive and value-based discussions in recent papers.

\section{NIME Eco Wiki}

The results of our summary review of the NIME proceedings are striking, if unsurprising. Out of nearly 2000 papers over 20 years of conferences, only twelve papers were identified that explicitly mentioned environmental issues. To support the NIME environmental statement, and encourage the community to fully engage with these important issues, we have initiated the NIME Eco Wiki which is introduced here.

In Interaction Design literature "the perspective that sustainability can and should be a central focus of interaction design" was introduced by Blevis [ㄴ5] , in the following discussion, environmental issues can be addressed from two perspectives (see for

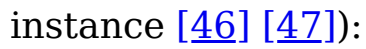

1. sustainability in design, when the environmental issues are addressed as part of the material design of an artefact

2. sustainability through design, when an artefact addresses environmental issues indirectly, focusing on "ways of living and processes of social change" [47].

The NIME Eco Wiki belongs to the second set of tools; it is a repository that aims to provide NIME practitioners with a collection of articles proposing solutions to environmental issues related to music technology or interaction design research in general.

In the following paragraphs, after describing the overall structure of the wiki, we discuss the governance and the wiki's intrinsic value against related literature on wikis and commons platforms.

\section{Overview}

The Eco Wiki has so far focused on three main points that, through initial discussions, emerged as potentially critical in fostering an environmentally sustainable practice. 
The first point aims to account for environmental issues in the overall work organisation, including workflow, communication, and online presence. The wiki pages that belong to this category are not specifically related to NIME but could be useful to support a more environmentally sustainable workflow generally. As office workflow can constitute a massive percentage of a NIME researcher's activity, we decided to give this topic an important place in the wiki. Entries about communication tools, video conferencing, and web hosting services belong to this category.

The second point addresses the development of new NIMEs. This is probably the wiki's central goal and the element that is more exclusive to the NIME community. Entries that fall in this category, so far, include 3D printing and machine learning.

Finally, the third point aims at collecting related projects and initiatives. In the last years, many artistic and music initiatives, as well as HCI projects related to the need to battle the effects of climate change have emerged. This page aims to collect such projects that can be of inspiration for supporting an environmentally aware NIME practice.

\section{Governance and dynamics in the Eco Wiki}

Up to this point, five persons directly contributed to the wiki, and the three proposers of the wiki have edited the main corpus of entries. This mirrors a general trend in wikis, as Arazy and colleges pointed out: "few highly active members and many occasional editors that make a few contributions"[48]. In light of this, there is still quite some work to do in order to render this wiki a common belonging to the community. In the following section, we outline some reflections about the work done so far.

\section{Information quality}

In their paper about information quality and work organisation in Wikipedia [49], Stvilia et al. outlined strategies that have been created to guarantee standards within Wikipedia. The authors outline how information quality tools and artefacts (e.g. templates, best practice guides) "are continually created to promote consistency in the content, structure, and presentation of articles" [49]. The authors also highlighted how the various entries are "carefully reviewed and discussed".

Following the same strategy, we developed a template to provide each page with a similar structure and support the reader in navigating and browsing through the different entries. In this template, we included a section where we ask any author of an 
entry to frame the topic with specific questions about environmental impact and possible solutions. We argue that this exercise can help reflect upon which perspectives are more relevant for that entry. Moreover, we created a list of possible entries to suggest additions to the wiki. We decided to softly curate the wiki to control the information quality and check the formatting. So far, the only edition performed on other authors' entries has been a reformatting of information to maintain coherence among the articles.

Arazy et al. analysed how Wikipedia group work is organised to assure information quality [48]. The authors, distinguished between two groups (administrative, and content creators) and different ways in which these groups impact the quality of the articles. We acknowledge that the Eco Wiki is too small to generate articulated interactions. However, the structure we have given to the wiki aims at supporting positive collaborations. For instance, having a list of possible needed entries can help diffuse the tasks, and providing the template can foster clarity in the content.

\section{Resembling FLOSS and implicit values}

O'Maho stated that "Wikipedia is arguably the first application of the open source community-managed model to a new context" [미]. Stvilia et al. highlighted how pages are "carefully reviewed and discussed in ways very similar to open source programming projects." [49]. Therefore, we also want to use the lens of FLOSS-related literature to look at the Eco Wiki.

In their book about values, commons, and computing, Hakken et al. argued that the core value of FLOSS and Wikipedias, is an implicit approach to openness [51]. The authors proposed that such openness affords excellent flexibility in facilitating collective decisions; therefore, it can represent collective wishes. Consequently, such a model can oppose the economic perspective generally valued in a centralised and capitalistic approach to computing. On the contrary, it can support the democratisation of knowledge and governance. Following a similar perspective, Poderi proposed the idea of "platforms as commons" to describe those platforms "based on various arrangements for the shared ownership and management of both their infrastructures and services or products" [52].

Based on these reflections, we believe that the Eco Wiki won't merely represent a tool to support a more environmentally aware NIME practice, but can also facilitate the emergence of shared values and reflection about this topic. Additionally, the wiki may support the first point proposed by Morreale et al. to develop an out-looking agenda 
for NIME community [프] : to deepen current conversations and start new ones. We hope that this tool can facilitate a debate within NIME, and soon involve many direct or indirect contributors and readers representing, therefore, multiple perspectives and collective wishes.

\section{Discussion}

\section{Current limitations of the NIME Eco Wiki}

The main limitation of the wiki so far is that it is still in its infancy. Being five months old and the number of people involved relatively low, the number of entries and related content is still limited. The main priority, therefore, is to attract more contributors. This will be done by letting more people know about the resource, and also develop a culture of contribution.

\section{Diversity}

One disadvantage of the wiki is that the current contributors are all white males. In her reflection about the type of knowledge in Wikipedia, van der Velden points out how the design process is ongoing but no longer dynamic or transformative [54]. This transformed Wikipedia from "a project with the potential to decolonize knowledge" to a new form of "hegemonic order" [4]. We acknowledge that this is a risk that the NIME Eco Wiki can face, and are eager to address it while the project grows, hopefully in collaboration with NIME's diversity and ethical officers.

\section{Language}

English is the lingua franca of international research communication, and the Eco Wiki also began as an English-language resource. This is a good way to reach the (currently) active NIME community, within which many non-native English speakers are also involved. Yet, we are aware of the limitations this put on the community's ability to grow beyond its current state. This is not intentional, and we acknowledge that the language may be a barrier to many possible contributors, and be seen as a, de facto, neocolonialist behaviour. Recent initiatives, such as the Helsinki Initiative on Multilingualism in Scholarly Communication [ㄷ5], promote language diversity in research. Some conferences, such as the Participatory_Design Conference (PDC) 2020 allowed authors to submit papers in the local languages (the conference was organized in South America, so Spanish and Portuguese were allowed). This attracted many local scholars, enriching the debate with a perspective that would otherwise be absent. 
In our experience, many undergraduate study programmes are typically taught in local languages, while it is more common to use English in graduate programmes. Having access to information in a local language could positively impact many students and practitioners. Just in the same way that Wikipedia has multilingual support, we would like to also facilitate the possibility of multiple languages in the Eco Wiki. Technically this is feasible, but we are unsure whether there is currently enough traction to support the translation of content. Developing and maintaining new content in a multilingual wiki is also much more time-consuming. One solution may be to first grow the English-language version of the wiki, and then plan an initiative for translation into other languages. This should be a community effort.

\section{A NIME sustainability framework}

In the following, we propose a matrix that can be thought of as a systematic approach to raise awareness and engagement around ecological and environmental issues in NIME research practices. It is intended to be high level and adaptable to the diversity of NIME. This includes research spanning the arts and humanities, social and natural sciences, and engineering. It also includes artistic practices and the variety of intermedial approaches in between science and art. The model is presented in the following table and consists of two primary axes: the research stage and its resources.

\begin{tabular}{|l|l|l|l|l|}
\hline & \multicolumn{2}{|c|}{ Research stage } & & \\
\hline Resources & Making & Testing & Using & Disposing \\
\hline & Consumables & & & \\
\hline Storage & & & \\
\hline & Processing & & & \\
\hline & Transport & & & \\
\hline
\end{tabular}

\section{Research stage}

The horizontal axis represents the stage of research. While arranged roughly chronologically, in practice research tends to overlap, iterate and move between the different stages in nonlinear ways. 
- Making: Covering the design, construction, prototyping, coding, etc. of NIMEs, in both physical and digital domains.

- Testing: Related to the testing of NIMEs, including in-progress rehearsals of new works.

- Using: Depending on the context, this can include performance and execution of artistic and musical works, utilising tools in scientific research, carrying out studies and experiments. This could also extend to the dissemination of results: traveling to conferences to give presentations, publishing, and similar activities.

- Disposing: Anything concerning end-of-life stages of a NIME (physical or digital).

The stages most closely represent the design cycle of a new NIME; however, it can be applied to most other research scenarios.

\section{Resources}

The vertical axis describes the basic categories of resources for which environmental impacts can be assessed and addressed. The actual items and steps towards environmental action will vary depending on the type of research practice.

- Materials: Physical or digital elements used in the construction of new instruments, tools, or other artefacts developed within the context of NIME research and practice.

- Consumables: Materials used in NIME research that are not part of an artefact.

- Storage: Infrastructures for the storage and archiving of research-related data and assets, including local and cloud-based digital storage solutions.

- Processing: Computational resources consumed in the carrying out of research, with consideration given to the energy sources used.

- Transport: Any resources related to transportation, most importantly commuting and travel for research, touring, conferences and other global events. It also includes the transport of materials and consumables.

The research stages and resources will significantly depend on the type of research practice, and not all stages and categories of resources will be present for all practices. However, we hope that most, if not all, NIME research activities can be mapped into the cells of this model and it can provide a framework for considering these topics in our day-to-day work.

We envision the NIME sustainability framework to complement the Eco Wiki. It can also serve as a way to organise the various stages and resource categories in the wiki with relevant information on specific research topics. 


\section{Conclusion}

The fragile state of the environment is well recognised as one of the most acute crises of our time. Based on numerous conversations and discussions throughout the NIME community, and spurred by the online organisation of the NIME 2020 conference due to the ongoing COVID-19 pandemic, we argue that careful consideration and direct action are necessary to address environmental issues in all facets of our research and creative practices.

In this paper, we provide an overview of how environmental sustainability and NIME practice intersect and lay out three primary contributions to the NIME community to prioritise environmental action in our collective work.

First, we investigated how environmental issues have been previously addressed in the NIME literature. Our systematic review of NIME proceedings revealed that active engagement with environmental issues in NIME research has been scarcely discussed: only 12 out of 1867 papers explicitly mention environmental topics.

Second, to address this lack of attention we have created the NIME Eco Wiki, a repository of information and resources on environmental issues for NIME researchers. It is in the early stages, but we invite the community to consult and contribute to the wiki. We hope that the resource can grow over time, and that the diversity of contributors and contributions will increase. Particular attention should be on implementing multilanguage support and ongoing collaboration with NIME's ethics and diversity initiatives.

Finally, we suggest a NIME sustainability framework for the systematic inclusion of environmental action in NIME research practice. The framework is based on identifying stages of research and categories of resources used, and aims to be broad enough to allow for the rich diversity of practices that are carried out under the NIME umbrella. In coordination with the Eco Wiki it can hopefully lead to the discovery and implementation of practical measures to lessen our work's ecological impact.

In conclusion, we offer this perspective from the NIME environmental statement:

As humans, we have a responsibility towards our environment and ecosystem. As artists and researchers, we have the ability to give voice to climate change and to confront and question damaging privileges and habits, through the development of new practices, technologies and infrastructures that lessen our planetary 
impact. As individuals, our impact is negligible, but as a community, we can make a difference.

\section{Acknowledgements}

This paper was written collaboratively by the three NIME environmental officers, along with the chair of the NIME Steering Committee. Authorship is attributed equally regardless of author order.

Raul Masu acknowledges ARDITI -Agencia Regionalpara o Desenvolvimento e Tecnologia under the scope of the Project M1420-09-5369-FSE-000002 - PhD Studentship for supporting his research.

Adam Pultz Melbye acknowledges the support of The Department for the Economics, Northern Ireland.

John Sullivan acknowledges the support of the Centre for Interdisciplinary Research in Music Media and Technology (CIRMMT).

Alexander Refsum Jensenius acknowledges the support of the Research Council of Norway through its Centres of Excellence scheme, project numbers 250698 and 262762.

\section{Compliance with Ethical Standards}

This paper complies with the NIME ethical standards, the authors do not see any ethical issue with this research and no human or animal participants are involved.

\section{Footnotes}

1. The results are available here:

https://gitlab.com/johnnyvenom/eco nime keywords $\triangleq$

\section{Citations}

1. Marquez-Borbon, Adnan, \& Stapleton, Paul. (2015). Fourteen Years of NIME: The Value and Meaning of 'Community' in Interactive Music Research. In Proceedings of the International Conference on New Interfaces for Musical Expression (pp. 307312). Baton Rouge, Louisiana, USA: Zenodo. http://doi.org/10.5281/zenodo.1179128 2. Cantrell, Joe. (2017). Designing Intent: Defining Critical Meaning for NIME Practitioners. In Proceedings of the International Conference on New Interfaces for 
Musical Expression (pp. 169-173). Copenhagen, Denmark: Zenodo. http://doi.org/10.5281/zenodo.1176211

3. Armitage, Jack, Morreale, Fabio, \& McPherson, Andrew. (2017). The finer the musician, the smaller the details: NIMEcraft under the microscope. In Proceedings of the International Conference on New Interfaces for Musical Expression (pp. 393398). Copenhagen, Denmark: Zenodo. http://doi.org/10.5281/zenodo.1176294

4. Magnusson, Thor, \& Mendieta, Enrike H. (2007). The Acoustic, the Digital and the Body : A Survey on Musical Instruments. In Proceedings of the International Conference on New Interfaces for Musical Expression (pp. 94-99). New York City, NY, United States: Zenodo. http://doi.org/10.5281/zenodo.1177171

5. Tanaka, Atau, Parkinson, Adam, Settel, Zack, \& Tahiroglu, Koray. (2012). A Survey and Thematic Analysis Approach as Input to the Design of Mobile Music GUIs. In Proceedings of the International Conference on New Interfaces for Musical Expression (p. x-x). Ann Arbor, Michigan: Zenodo. http://doi.org/10.5281/zenodo.1178431 6. Morreale, Fabio, McPherson, Andrew P., \& Wanderley, Marcelo. (2018). NIME Identity from the Performer's Perspective. In Proceedings of the International Conference on New Interfaces for Musical Expression (pp. 168-173). Blacksburg, Virginia, USA: Zenodo. http://doi.org/10.5281/zenodo.1302533 7. Jensenius, Alexander Refsum. (2014). To gesture or Not? An Analysis of Terminology in NIME Proceedings 2001-2013. In Proceedings of the International Conference on New Interfaces for Musical Expression (pp. 217-220). London, United Kingdom: Zenodo. http://doi.org/10.5281/zenodo.1178816

8. Barbosa, Jeronimo, Malloch, Joseph, Wanderley, Marcelo, \& Huot, Stéphane. (2015). What does 'Evaluation' mean for the NIME community?. In Proceedings of the International Conference on New Interfaces for Musical Expression (pp. 156161). Baton Rouge, Louisiana, USA: Zenodo. http://doi.org/10.5281/zenodo.1179010 9. Refsum Jensenius, Alexander, and Michael J. Lyons. "Trends at NIME--Reflections on Editing" A NIME Reader"." arXiv e-prints (2020): arXiv-2010. $\subseteq$ 10. Sullivan, J., \& Wanderley, M. M. (2018). Stability, Reliability, Compatibility: Reviewing 40 Years of DMI Design. In Proceedings of the 15th Sound and Music Computing Conference (SMC) (pp. 319-326). Limassol, Cyprus. $\_$ 
11. Gurevich, Michael, \& Trevi no, Jeffrey. (2007). Expression and Its Discontents : Toward an Ecology of Musical Creation. In Proceedings of the International Conference on New Interfaces for Musical Expression (pp. 106-111). New York City, NY, United States: Zenodo. http://doi.org/10.5281/zenodo.1177107

12. Waters, Simon. "Performance Ecosystems: Ecological approaches to musical interaction." EMS: Electroacoustic Music Studies Network (2007): 1-20. 13. Avila, J. P. M., Greenhalgh, C., Hazzard, A., Benford, S., \& Chamberlain, A. (2019). Encumbered interaction: A study of musicians preparing to perform. In Proceedings of the 2019 CHI Conference on Human Factors in Computing Systems (pp. 1-13). $\doteq$

14. Masu, R., Correia, N. N., Jurgens, S., Feitsch, J., \& Rom ao, Teresa. (2020). Designing Interactive Sonic Artefacts for Dance Performance: An Ecological Approach. In Proceedings of the 15th International Conference on Audio Mostly (pp. 122-129). Graz, Austria: Association for Computing Machinery. https://doi.org/10.1145/3411109.3412297 $ヒ$ 15. Masu, R., Bettega, M., Correia, N. N., Rom ao, Teresa, \& Morreale, F. (2019). ARCAA: A Framework to Analyse the Artefact Ecology in Computer Music Performance. In Proceedings of the 9th International Conference on Digital and Interactive Arts. Braga, Portugal: Association for Computing Machinery. https://doi.org/10.1145/3359852.3359870 16. Rodger, M., Stapleton, P., van Walstijn, M., Ortiz, M., \& Pardue, L. S. (2020). What Makes a Good Musical Instrument? A Matter of Processes, Ecologies and Specificities. In Proceedings of the International Conference on New Interfaces for Musical Expression (pp. 484-490). Birmingham, UK. $\triangleq$

17. Hayes, Lauren, and Adnan Marquez-Borbon. "Nuanced and Interrelated Mediations and Exigencies (NIME): Addressing the Prevailing Political and Epistemological Crises." In Proceedings of the International Conference on New Interfaces for Musical Expression $2020 \subseteq$

18. Morreale, Fabio, et al. "A NIME of the Times: Developing an Outward-Looking Political Agenda For This Community." New Interfaces for Musical Expression. 2020.

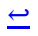

19. Barrie Heaton, Keys and Keyboards https://www.pianotuners.org/history/compass.html $€$ 
20. Lemieux, A. M., \& Clarke, R. V. (2009). The International Ban on Ivory Sales and its Effects on Elephant Poaching in Africa. The British Journal of Criminology, 49(4), 451-471. https://doi.org/10.1093/bje/azp030

21. https://www.guitarplayer.com/gear/whats-the-big-deal-about-brazilian-rosewood $\triangleq$ 22. Devine, K. (2019). Decomposed: The Political Ecology of Music. MIT Press. $ヒ$ 23. Collins, N. (2020). Salvage (2008) and The Royal Touch (2014). Echo: A Journal of Music, Thought and Technology, (1). https://doi.org/10.2307/j.ctv1198z4v.33

24. https://enviroliteracy.org/special-features/its-element-ary/silicon/ $\triangleq$

25. https://eco.nime.org/entries/3d_printing_pla $ヒ$

26. Aizen, M. A., Aguiar, S., Biesmeijer, J. C., Garibaldi, L. A., Inouye, D. W., Jung, C., ... Seymour, C. L. (2019). Global agricultural productivity is threatened by increasing pollinator dependence without a parallel increase in crop diversification. Global Change Biology, 25(10), 3516-3527. https://doi.org/https://doi.org/10.1111/gcb.14736 27. https://www.musicradar.com/news/what-is-machine-learning-and-what-does-it$\underline{\text { mean-for-music }} \subseteq$

28. Fiebrink, R. (2016). Machine learning as meta-instrument: Human-machine partnerships shaping expressive instrumental creation. Musical Instruments in the 21st Century: Identities, Configurations, Practices, (1), 137-151. https://doi.org/10.1007/978-981-10-2951-6 $10 \subseteq$ 29. Strubell, E., Ganesh, A., \& McCallum, A. (2019). Energy and policy considerations for deep learning in NLP. ArXiv, (1), 3645-3650. 30. Kiefer, C., Overholt, D., \& Eldridge, A. (2020). Shaping the behaviour of feedback instruments with complexity-controlled gain dynamics. In R. Michon \& F. Schroeder (Eds.), Proceedings of the International Conference on New Interfaces for Musical Expression (pp. 343-348). Birmingham, UK: Birmingham City University. Retrieved from https://www.nime.org/proceedings/2020/nime2020_paper66.pdf 31. Berdahl, E., \& Ju, W. (2011). Satellite CCRMA: A Musical Interaction and Sound Synthesis Platform. In Proceedings of the International Conference on New 
Interfaces for Musical Expression (pp. 173-178). Oslo, Norway. Retrieved from http://www.nime.org/proceedings/2011/nime2011\173.pdf

32. Marquez-Borbon, Adnan, \& Martinez-Avila, Juan Pablo. (2018). The Problem of DMI Adoption and Longevity: Envisioning a NIME Performance Pedagogy. In Proceedings of the International Conference on New Interfaces for Musical Expression (pp. 190-195). Blacksburg, Virginia, USA: Zenodo. http://doi.org/10.5281/zenodo.1302541 33. Morreale, F., \& McPherson, A. (2017). Design for Longevity: Ongoing Use of Instruments from NIME 2010-14. In Proceedings of the International Conference on New Interfaces for Musical Expression (pp. 192-197). Copenhagen, Denmark: Aalborg University Copenhagen. https://doi.org/10.5281/zenodo.1176218 34. Suchánek, J. (2020). SOIL CHOIR v.1.3 - Soil Moisture Sonification Installation. In R. Michon \& F. Schroeder (Eds.), Proceedings of the International Conference on New Interfaces for Musical Expression (pp. 738-740). Birmingham, UK: Birmingham City University. $€$ 35. Magnusson, T. (2020). Instrumental Investigations at Emute Lab. In R. Michon \& F. Schroeder (Eds.), Proceedings of the International Conference on New Interfaces for Musical Expression (pp. 509-513). Birmingham, UK: Birmingham City University. Retrieved from https://www.nime.org/proceedings/2020/nime2020_paper97.pdf $\leftrightarrows$ 36. Lynch, Evan, \& Paradiso, Joseph. (2016). SensorChimes: Musical Mapping for Sensor Networks. In Proceedings of the International Conference on New Interfaces for Musical Expression (pp. 137-142). Brisbane, Australia: Zenodo. http://doi.org/10.5281/zenodo.1176074 37. Smallwood, Scott. (2011). Solar Sound Arts: Creating Instruments and Devices Powered by Photovoltaic Technologies. In Proceedings of the International Conference on New Interfaces for Musical Expression (pp. 28-31). Oslo, Norway: Zenodo. http://doi.org/10.5281/zenodo.1178167 38. Gaye, Lalya, \& Holmquist, Lars E. (2004). In Duet with Everyday Urban Settings: A User Study of Sonic City. In Proceedings of the International Conference on New Interfaces for Musical Expression (pp. 161-164). Hamamatsu, Japan: Zenodo. http://doi.org/10.5281/zenodo.1176601 39.

Gaye, Lalya, Mazé, Ramia, \& Holmquist, Lars E. (2003). Sonic City: The Urban Environment as a Musical Interface. In Proceedings of the International Conference on New Interfaces for Musical Expression (pp. 109-115). Montreal, Canada: Zenodo. http://doi.org/10.5281/zenodo.1176507 


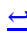

40. Alexandra Rieger and Spencer Topel. 2016. Driftwood: Redefining Sound Sculpture Controllers. Proceedings of the International Conference on New Interfaces for Musical Expression, Queensland Conservatorium Griffith University, pp. 158-159. http://doi.org/10.5281/zenodo.1176110

41. Adrian Freed. 2012. The Fingerphone: a Case Study of Sustainable Instrument Redesign. Proceedings of the International Conference on New Interfaces for Musical Expression, University of Michigan. http://doi.org/10.5281/zenodo.1178253 42. Koehly, Rodolphe, Curtil, Denis, \& Wanderley, Marcelo M. (2006). Paper FSRs and Latex/Fabric Traction Sensors: Methods for the Development of Home-Made Touch Sensors. In Proceedings of the International Conference on New Interfaces for Musical Expression (pp. 230-233). Paris, France $\leftrightharpoons$

43. Fraietta, Angelo. (2008). Open Sound Control : Constraints and Limitations. In Proceedings of the International Conference on New Interfaces for Musical Expression (pp. 19-23). Genoa, Italy: Zenodo. http://doi.org/10.5281/zenodo.1179537

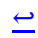

44. Lepri, Giacomo, \& McPherson, Andrew P. (2019). Fictional instruments, real values: discovering musical backgrounds with non-functional prototypes. In Proceedings of the International Conference on New Interfaces for Musical Expression (pp. 122-127). Porto Alegre, Brazil: Zenodo. http://doi.org/10.5281/zenodo.3672890 45. Blevis, E. (2007). Sustainable interaction design: invention \&disposal, renewal \&reuse. In Proceedings of the SIGCHI conference on Human factors in computing systems (pp. 503-512). $\subseteq$

46. Mankoff, J. C., Blevis, E., Borning, A., Friedman, B., Fussell, S. R., Hasbrouck, J., ... Sengers, P. (2007). Environmental Sustainability and Interaction. In $\mathrm{CHI}$ '07 Extended Abstracts on Human Factors in Computing Systems (pp. 2121-2124). San Jose, CA, USA: Association for Computing Machinery. https://doi.org/10.1145/1240866.1240963 47. Morley, J. (2017). Response 1b: Sustainability within HCI within society: improvisations, interconnections and imaginations. 
48. Arazy, Ofer, Nov, O., Patterson, R., \& Lisa Yeo . (2011). Information Quality in Wikipedia: The Effects of Group Composition and Task Conflict. Journal of Management Information Systems, 27(4), 71-98. https://doi.org/10.2753/MIS0742$\underline{1222270403} \subseteq$

49. Stvilia, B., Twidale, M. B., Smith, L. C., \& Gasser, L. (2008). Information quality work organization in Wikipedia. Journal of the American Society for Information Science and Technology, 59(6), 983-1001.

50. O'Mahony, S. (2007). The governance of open source initiatives: what does it mean to be community managed? Journal of Management \&Governance, 11(2), 139150.

51. Hakken, D., Teli, M., \& Andrews, B. (2015). Beyond Capital: Values, Commons, Computing, and the Search for a Viable Future. Routledge. $\triangleq$

52. Poderi, G. (2019). Sustaining platforms as commons: perspectives on participation, infrastructure, and governance. CoDesign, 15(3), 243-255.

53. Morreale, F., Bin, A., McPherson, A., Stapleton, P., \& Wanderley, M. (2020). A NIME of the Times: Developing an Outward-Looking Political Agenda For This Community. In New Interfaces for Musical Expression. $ヒ$

54. Van der Velden, M. (2013). Decentering Design: Wikipedia and Indigenous Knowledge. International Journal of Human-Computer Interaction, 29(4), 308-316. https://doi.org/10.1080/10447318.2013.765768

55. Helsinki Initiative on Multilingualism in Scholarly Communication (2019). Helsinki: Federation of Finnish Learned Societies, Committee for Public Information, Finnish Association for Scholarly Publishing, Universities Norway \& European Network for Research Evaluation in the Social Sciences and the Humanities. https://doi.org/10.6084/m9.figshare.7887059.. 